J. Lake Sci.(湖泊科学), 2016, 28(3): 645-653

DOI 10. 18307/2016. 0321

(c) 2016 by Journal of Lake Sciences

\title{
HJ-1B/IRS 热红外数据反演太湖水温的方法比较
}

\author{
龚绍琦 ${ }^{1}$, 陆品廷 ${ }^{1}$, 孙德勇 ${ }^{1}$, 李云梅 ${ }^{2}$, 吴鹏飞 ${ }^{1}$ \\ ( 1 : 南京信息工程大学地理与遥感学院,南京 210044) \\ (2:江苏省地理信息资源开发与利用协同创新中心,南京 210023)
}

\begin{abstract}
摘 要: 太湖是我国典型的富营养化湖泊,水温是影响太湖藻类生长的重要环境因子之一, 我国环境减灾卫星 $\mathrm{HJ}-1 \mathrm{~B}$ 搭 载的红外多光谱相机 IRS 对太湖水温动态遥感监测具有较大的性能优势. 利用 6 景过境太湖的 IRS 热红外遥感影像, 分 别采用单通道普适性算法、辐射传输模型法和单窗算法反演太湖水温, 并与实测水温和同期的 TERRA/MODIS 温度产品 进行对比. 结果表明, 普适性单通道算法反演水温偏高, 而辐射传输模型法和单窗算法则偏低; 3 种算法反演水温的均方 根误差在 $1.001 \mathrm{~K}$ 以内, 单窗算法反演精度最高, 其次是辐射传输模型法, 再次为普适性单通道算法, 而同期 MODIS 温度 产品的均方根误差为 $1.507 \mathrm{~K} .3$ 种算法从 IRS 热红外数据反演的水温直方图均呈正峰态、尖峰状态分布, 反演结果能真 实地反映太湖水温的空间分布特征. 本研究对只有单个热红外通道的卫星传感器开展内陆水体水温遥感监测具有一定 的参考意义.
\end{abstract}

关键词: 太湖;水温;HJ-1B/IRS;热红外数据;算法

\section{Comparison of water surface temperature retrieval algorithms from $\mathrm{HJ}-1 \mathrm{~B} / \mathrm{IRS}$ thermal in- frared data over Lake Taihu, China}

GONG Shaoqi ${ }^{1}$, LU Pinting ${ }^{1}$, SUN Deyong ${ }^{1}$, LI Yunmei ${ }^{2}$ \& WU Pengfei ${ }^{1}$

(1: School of Geography and Remote Sensing, Nanjing University of Information Science and Technology, Nanjing 210044, P.R.China)

(2: Jiangsu Center for Collaboration Innovation in Geographical Information Resource Development and Application, Nanjing 210023, P.R.China)

Abstract: Lake Taihu is a typical eutrophic lake in China, and water surface temperature (WST) becomes one of the most important environmental factors for algae growing in Lake Taihu. The infrared scanner on board Chinese environment and disaster monitoring satellite HJ-1B has a good capability to monitor periodically WST by remote sensing technique. Generalized single-channel method (GSCM), radiative transfer code method (RTM) and mono-window algorithm (MWA) are applied to retrieve WST from six IRS thermal infrared images passing over Lake Taihu, then the retrieved WST is compared with measured one and TERRA/MODIS temperature product. The results show that the WST retrieved by GSCM is a little higher than measured one while that by RTM and WMA is a bit lower, in which the root mean square error ( RMSE) of WST calculated by three algorithms is within $1.001 \mathrm{~K}$. The accuracy of WMA is at the best, RTM is at the second good and the third good for GSCM. The RMSE of MODIS temperature product is $1.507 \mathrm{~K}$. The WST histograms retrieved from IRS thermal infrared data by three algorithms showed a positive deviation and a steep normal distribution, suggesting that WST thematic map can display correctly the spatial variability of temperature in Lake Taihu. This will play a guide role to carry out WST monitoring in inland waters by sensor which has only one thermal infrared channel on board satellite.

Keywords: Lake Taihu; water surface temperature; HJ-1B/IRS; thermal infrared data; algorithm

* 国家自然科学基金项目 (40801145,41576172)、江苏省自然科学基金项目 (BK20151526)、江苏高校优势学科建设 工程资助项目和高分辨率对地观测系统重大专项(32-Y30B08-9001-13) 联合资助. 2015-07-31 收稿; 2015-0831 收修改稿. 龚绍琦(1979 ), 男,博士, 副教授;E-mail: shaoqigong@163.com. 
太湖是我国第三大淡水湖, 不仅是国内外享有盛名的旅游胜地, 而且是上海、无锡、苏州等地的主要水 源, 兼有渔业、灌溉、航运等多种功能. 自 1980s 起, 太湖的富营养化程度越来越严重, 目前全湖处于富营养到 重富营养状态, 湖泊水质则属于劣 $\mathrm{V}$ 类 ${ }^{[1]}$. 太湖的富营养化导致蓝藻水华现象不断暴发, 而且水华面积有逐 年扩大、持续时间越来越长的趋势 ${ }^{[2]}$. 太湖蓝藻水华不仅破坏水体景观和生态系统平衡, 蓝藻在生长过程中 会释放毒素, 消耗水中溶解氧, 引起水生生物大量死亡, 湖泊水质恶化, 严重威胁了湖泊周围地区的饮水安 全 $^{[3]}$. 众多的研究表明太湖藻类数量与水温有很好的相关关系 ${ }^{[48]}$, 由于太湖富营养化为蓝藻生长提供了充 足的营养盐, 水温成了影响太湖藻类生长的重要环境因子之一, 全面有效地监测太湖水温对蓝藻水华的预 警预报具有一定的作用. 而卫星热红外遥感技术为太湖水温监测提供了一种高效快捷的手段, 目前 NOAA、 TERRA、AQUA、NPP、METOP、FY 等系列卫星都能获得相应的热红外遥感数据, 其时间分辨率高, 但空间分 辨率低, 难以满足内陆湖泊水温遥感监测; 而 Landsat 系列卫星的 TM/ETM+/TIRS 数据、TERRA 卫星的 ASTER 数据具有较好的空间分辨率, 比较适合内陆湖泊水温遥感监测, 但卫星观测周期为 $16 \mathrm{~d}$, 不利于水温动 态监测. 我国 2008 年 9 月 6 日发射的环境减灾卫星 HJ-1B 上搭载了一台红外多光谱相机 IRS, 具有 2 个近 红外通道、 1 个中红外通道和 1 个热红外通道, 空间分辨率分别为 $150 、 150$ 和 $300 \mathrm{~m}$, 对地观测周期为 $4 \mathrm{~d}$, 能 够为太湖水温动态遥感监测提供很好的资料. 目前, 国内学者针对 HJ-1B/IRS 单通道的热红外资料在遥感 算法改进、地表温度和水表面温度反演方面进行了一些研究 ${ }^{[9-13]}$, 由于像 HJ-1B/IRS 这种只带一个热红外通 道的卫星传感器仍有不少, 如 Nimbus-7/CZCS、Landsat-7/ETM+、CERBS/IRMSS、FY-3/MERSI, 因此很有必要 开展基于单通道热红外数据的遥感算法研究. 本文将针对 $\mathrm{HJ}-1 \mathrm{~B} / \mathrm{IRS}$ 单通道的热红外数据, 分别采用普适 性单通道算法 (generalized single-channel method, GSCM) ${ }^{[14]}$ 、辐射传输模型法 (radiative transfer code method, RTM) 和单窗算法 (mono-window algorithm, MWA $)^{[15]}$ 反演太湖水温, 比较 3 种反演方法的精度, 以期为 HJ$1 \mathrm{~B} / \mathrm{IRS}$ 资料在太湖水温遥感监测中的业务化应用提供理论依据.

\section{1 数据与方法}

\section{1 数据}

2008 年 11 月 $10-21$ 日和 2009 年 4 月 16- 27 日在太湖开展了水体光学实验, 测定水质参数、水体光 谱、水面气象参数等, 其中水表面温度用水温表测量, 精度为 $0.2 \mathrm{~K}$. 实验期间获得太湖地区有效的 HJ-1B/ IRS 遥感影像 6 景, 日期为 2008 年 11 月 10 日和 14 日, 2009 年 4 月 17 日、21 日、22 日和 25 日,影像质量好、 无云彩, 数据为 LEVEL 2 级产品, 下载于中国资源卫星应用中心. 在 HJ-1B 卫星过境时同步实测 7 个样点的 水温 (表 1 和图 1), 两者观测时间相差不到 $30 \mathrm{~min}$, 可以用于 IRS 热红外数据反演水温的精度验证. 表 1 中 HJ-1B 过境时间和观测天顶角从 IRS 影像的数据文件中获得, 卫星过境时的能见度是由位于太湖的东山气 象站 (58358) 在格林尼治时间 (GMT) 0:00 时和 6:00 时的观测值内插得到, 水汽量是来自全球气溶胶 Aeronet 网太湖站实测值, 大气平均作用温度 $T_{\mathrm{a}}$ 是由太湖周边气象站—上海站 (58362)、南京站 (58238) 和 杭州站 (58457) 在 0:00 时 (GMT) 的探空资料计算的平均值. 为了进一步说明 IRS 热红外数据反演水温的精 度, 引人了 TERRA/MODIS 温度产品进行对比, 同期的 TERRA 卫星过境时间如表 1 所示, 数据从 NASA 官网 http://reverb.echo.nasa.gov/reverb/下载得到.

\subsection{HJ-1B/IRS 热红外遥感影像的预处理}

(1) 遥感影像的辐射校正: 由于从中国资源卫星中心下载的 HJ-1B/IRS 影像资料已经做过几何校正, 满 足研究的需要. 这里只对 $\mathrm{HJ}-1 \mathrm{~B} / \mathrm{IRS}$ 热红外通道 (第 4 通道) 进行辐射校正, 将原始的数字信号 $D N$ 值转化 为图像辐亮度. 根据 IRS 影像自述文件中提供的辐射定标系数 $b$ 和 $g$, 按式(1)进行辐射校正:

$$
L=\frac{D N-b}{g}
$$

式中, $g=59.421 \mathrm{DN} /\left(\mathrm{W} /\left(\mathrm{m}^{2} \cdot \mathrm{sr} \cdot \mu \mathrm{m}\right)\right), b=-25.441$. 利用遥感图像处理软件 $\mathrm{ENVI}$ 进行辐射校正, 从而计 算出辐亮度值 $L$.

(2) 遥感影像亮度温度的计算: 根据普朗克定律计算亮度温度 $T_{\mathrm{B}}$ : 
表 $1 \mathrm{HJ}-1 \mathrm{~B}$ 卫星过境太湖时卫星、气象和水温的信息

Tab.1 Parameters of satellite, meteorology and water surface temperature when HJ-1B passing over Lake Taihu

\begin{tabular}{lcccccccccc}
\hline 日期 & $\begin{array}{c}\mathrm{HJ}-1 \mathrm{~B} / \\
\mathrm{GMT}\end{array}$ & $\begin{array}{c}\text { 观测天 } \\
\text { 顶角 }^{\circ}\end{array}$ & $\begin{array}{c}\text { 能见度/ } \\
\mathrm{km}\end{array}$ & $\begin{array}{c}\text { 水汽量/ } \\
\mathrm{cm}\end{array}$ & $\begin{array}{c}\text { 大气平均 } \\
\text { 作用温度/K }\end{array}$ & GMTRA/ & $\begin{array}{c}\text { 水温实测 } \\
\text { 时间/GMT }\end{array}$ & 纬度 & 经度 & $\begin{array}{c}\text { 水温/ } \\
\mathrm{K}\end{array}$ \\
\hline $2008-11-10$ & $3: 02$ & 23.3 & 22.3 & 0.924 & 275.9 & $3: 35$ & $3: 16$ & 31.487 & 120.217 & 287.8 \\
$2008-11-14$ & $3: 05$ & 20.7 & 10.0 & 1.77 & 281.9 & $3: 10$ & $2: 56$ & 31.151 & 120.000 & 288.8 \\
$2009-04-17$ & $2: 35$ & 27.8 & 14.0 & 1.65 & 279.2 & $2: 10$ & $2: 15$ & 31.414 & 120.324 & 291.2 \\
$2009-04-21$ & $2: 38$ & 20.0 & 14.5 & 1.19 & 284.4 & $3: 00$ & $2: 10$ & 31.442 & 120.198 & 292.3 \\
& & & & & & & $2: 50$ & 31.417 & 120.134 & 292.6 \\
$2009-04-22$ & $3: 03$ & 23.7 & 10.5 & 1.774 & 280.3 & $2: 30$ & $2: 11$ & 31.366 & 120.006 & 294.6 \\
$2009-04-25$ & $2: 41$ & 18.4 & 14.5 & 1.456 & 279.1 & $3: 00$ & $2: 58$ & 31.260 & 119.960 & 292.5 \\
\hline
\end{tabular}

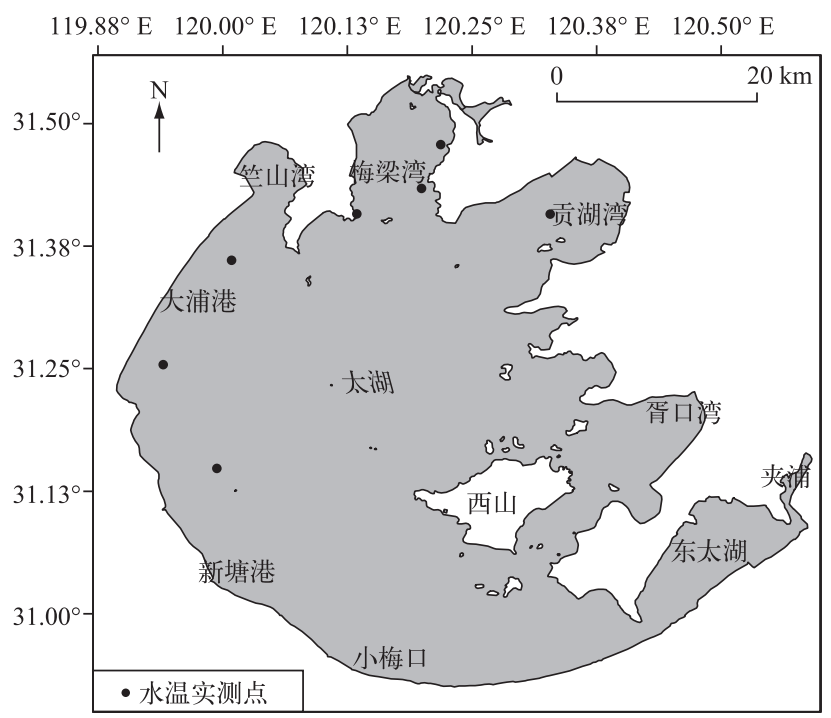

图 1 太湖水温实测点的位置

Fig.1 Location of measured water surface temperature (WST) sites in Lake Taihu

$$
T_{\text {B }}=\frac{C_{2}}{\lambda \cdot \ln \left(1+\frac{C_{1}}{\lambda^{5} \cdot L}\right)}
$$

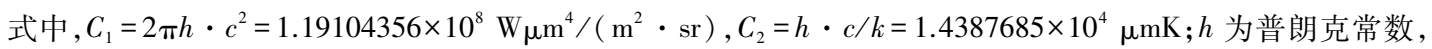
取 $6.626 \times 10^{-34} \mathrm{~J} \cdot \mathrm{s} ; c$ 为真空中的光速, 取 $2.998 \times 10^{8} \mathrm{~m} / \mathrm{s} ; k$ 为波耳茨曼常数, 取 $1.38 \times 10^{-23} \mathrm{~J} / \mathrm{K} ; \lambda$ 为 $\mathrm{HJ}-1 \mathrm{~B} /$ IRS 热红外通道的中心波长, $\lambda=11.576 \mu \mathrm{m}, L$ 是辐亮度值, 经式(2)计算便可得到遥感影像的亮度温度.

\section{3 研究方法}

根据热辐射传输原理, 当热红外传感器观测湖面时, 人瞳辐亮度 $L_{\mathrm{t}}$ 有 3 个来源 ${ }^{\left[{ }^{16]}\right.}$ : (1)水面的热红外辐射 $L_{\mathrm{w}}$, 这部分的能量取决于水面温度、水的发射率 $\varepsilon$ 以及水面和传感器之间的大气传输路径中的透过率 $\tau$; (2)大气自身发射的下行热辐射 $L_{\mathrm{a}}^{\downarrow}$, 到达水面后, 经水面反射并穿过大气层, 被传感器所接收的辐射; (3)气 自身发射的上行热辐射 $L_{\mathrm{a}}^{\uparrow}$, 直接进人传感器的辐射. 研究表明, 大气自身发射的热辐射与大气中吸收气体 的含量和物理状态有关, 主要的影响因素为大气温度和水汽量 ${ }^{[14-15]}$. 因此, 传感器接收到的总人睲辐亮度 $L_{\mathrm{t}}$ 可表示为: 


$$
L_{\mathrm{t}}=\varepsilon \cdot \tau \cdot L_{\mathrm{w}}+(1-\varepsilon) \tau \cdot L_{\mathrm{a}}^{\downarrow}+L_{\mathrm{a}}^{\uparrow}
$$

由于水的发射率高, 近似为黑体, 水面反射率 $1-\varepsilon$ 接近为 0 , 因此经水面反射的大气下行热辐射 $(1-$ $e) t L_{\mathrm{a}}$ 部分可忽略,式 (3) 则简化为:

$$
L_{\mathrm{t}}=\varepsilon \cdot \tau \cdot L_{\mathrm{w}}+L_{\mathrm{a}}^{\uparrow}
$$

式中, $L_{t}$ 是遥感传感器接收的总热辐射; $\varepsilon$ 是湖水的发射率, 由于太湖是淡水湖泊, 而相同温度下发射率主要 取决于物质的自身组成, 根据 ASTER 光谱库 (http://speclib.jpl. nasa.gov/) 中水实测的光谱数据, 结合 IRS 热红外通道的光谱响应函数进行卷积, 得到 IRS 热红外通道水的发射率为 $0.9871 ; \tau$ 是水面和传感器之间大 气传输路径中的透过率, 主要受卫星观测几何和大气成分的影响, 一般可用辐射传输模型模拟 ${ }^{[17]}$ 或经验公 式 ${ }^{[18-19]}$ 计算得到; $L_{w}$ 是在一定温度下水面当作黑体时发射的热辐射, 是温度遥感反演中待求解的物理量; 在 温度遥感反演算法中, 大气自身发射的上行热辐射 $L_{\mathrm{a}}^{\uparrow}$ 一般通过对普朗克函数运用泰勒展开式进行线性近 似, 最终转化为大气平均作用温度或水汽量的函数 ${ }^{[14-15]}$. 因此, 根据式 (4) 本文将采用普适性单通道算法、辐 射传输模型法和单窗算法从 IRS 热红外数据中反演太湖水温, 并对反演算法进行精度比较.

表 2 辐射传输模型 MODTRAN 模拟的 HJ/IRS 大气参数

Tab.2 Atmospheric parameters simulated by radiative transfer code MODTRAN

\begin{tabular}{ccc}
\hline 日期 & $\begin{array}{c}\text { 大气上行辐射/ } \\
\left(\mathrm{W} /\left(\mathrm{m}^{2} \cdot \mathrm{sr} \cdot \mu \mathrm{m}\right)\right)\end{array}$ & 大气透过率 \\
\hline $2008-11-10$ & 1.1014 & 0.822 \\
$2008-11-14$ & 2.0563 & 0.718 \\
$2009-04-17$ & 1.8397 & 0.735 \\
$2009-04-21$ & 1.5555 & 0.805 \\
$2009-04-22$ & 2.1801 & 0.697 \\
$2009-04-25$ & 1.707 & 0.749 \\
\hline
\end{tabular}

1.3.1 辐射传输模型法 由式(4)可见,要从卫星 热红外遥感图像中提取水面发射的热辐射 $L_{\mathrm{w}}$, 首 先要确定大气透过率 $\tau$ 和大气自身发射的上行 热辐射 $L_{\mathrm{a}}^{\uparrow}$. 因此, 可利用辐射传输模型 MODTRAN 4.0 模拟 IRS 热红外通道的大气透过 率和大气上行热辐射, 模拟时的大气模型 2008 年 11 月为中纬冬季、2009 年 4 月为中纬夏季, 气 溶胶模型为城市型,所需的观测天顶角和气象参 数见表 1 . 模拟结果与 IRS 光谱响应函数进行卷 积运算, 得到 IRS 热红外通道的大气透过率和大 气上行热辐射 (表 2). 从 IRS 热红外图像中可计 算出水面发射的热辐射 $L_{\mathrm{w}}$, 再根据普朗克函数由 $L_{\mathrm{w}}$ 计算出太湖水温.

1.3.2 单窗算法 覃志豪等针对 Landsat/TM6 数据构建了反演地表温度的单窗算法 ${ }^{[15]}$, 由于本研究是针对 水体, 在忽略水面反射的大气下行热辐射后, 需对原算法进行重新推导, 得到反演水温 $T_{w}$ 的单窗算法:

$$
T_{\mathrm{w}}=\left\{a \cdot \tau(1-\varepsilon)+[1-(1-b)(1-\varepsilon) \tau] T_{\mathrm{b}}-(1-\tau) T_{\mathrm{a}}\right\} /(\varepsilon \cdot \tau)
$$

式中, $a$ 和 $b$ 是单窗算法中定义的温度参数 $L=B(T) /[B(T) / \partial T]$ 与温度 $T$ 之间的线性回归系数, $B(T)$ 为 普朗克函数, IRS 热红外通道中心波长为 $11.567 \mu \mathrm{m}$, 在 $-5 \sim 45^{\circ} \mathrm{C}$ 时 $a=-62.360, b=0.4395$, 回归决定系数 $R^{2}=$ 0.999 , 均方根误差 $R M S E=0.1206 ; T_{\mathrm{b}}$ 是遥感图像的亮度温度; $T_{\mathrm{a}}$ 是大气平均作用温度 (表 1$) ; \tau$ 是大气透射 率, 可由辐射传输模型 MODTRAN 模拟得到 (表 2) $; \varepsilon$ 是湖水的发射率.

1.3.3 普适性单通道算法 Jiménez-Muñoz 等建立了从遥感数据中反演地表温度的普适性单通道算法 ${ }^{[14]}$, 对 于海表面温度 SST 的反演,算法简化为:

$$
T_{\mathrm{SST}} \approx \gamma\left(\lambda, T_{0}\right)\left[\psi_{1}^{S S T}(\lambda, w) L_{\lambda}^{\mathrm{at}-\text {-sensor }}+\psi_{2}^{S S T}(\lambda, w)\right]+\delta\left(\lambda, T_{0}\right)
$$

式中, $L$ 是大气顶层传感器接收的热辐射亮度; $\gamma$ 和 $\delta$ 是热辐射亮度与温度之间按照泰勒线性近似展开的函 数, $\gamma\left(\lambda, T_{0}\right) \equiv \frac{1}{\beta\left(\lambda, T_{0}\right)}, \delta\left(\lambda, T_{0}\right) \equiv-\frac{\alpha\left(\lambda, T_{0}\right)}{\beta\left(\lambda, T_{0}\right)}$, 而 $\alpha$ 和 $\beta$ 则表示为:

$$
\begin{gathered}
\alpha\left(\lambda, T_{0}\right) \equiv B\left(\lambda, T_{0}\right)\left[1-\frac{c_{2}}{T_{0}}\left(\frac{\lambda^{4}}{c_{1}} B\left(\lambda, T_{0}\right)+\frac{1}{\lambda}\right)\right] \\
\beta\left(\lambda, T_{0}\right) \equiv \frac{c_{2} B\left(\lambda, T_{0}\right)}{T_{0}^{2}}\left[\frac{\lambda^{4}}{c_{1}} B\left(\lambda, T_{0}\right)+\frac{1}{\lambda}\right]
\end{gathered}
$$

式中, $T_{0}$ 可看作是卫星传感器的亮度温度; $B$ 为普朗克函数; $\psi_{1}$ 和 $\psi_{2}$ 为大气参数, 在热红外波段范围可用大 气水汽量 $w$ 来表示. 对于 IRS 热红外通道, $\psi_{1}$ 和 $\psi_{2}$ 的计算表达式为: 


$$
\begin{gathered}
\psi_{1}=0.024764 w^{3}-0.031750 w^{2}+0.186992 w+0.993281 \\
\psi_{2}=-0.230743 w^{3}+0.255181 w^{2}-1.283163 w+0.211181
\end{gathered}
$$

\section{2 结果与分析}

\section{1 太湖水温反演结果的比较}

根据前述的 3 种反演算法, 分别从 6 景 IRS 热红外 遥感图像中反演太湖水温. 根据水温实测点的经纬度坐 标, 从反演结果中提取相应的水温值, 并与实测水温进行 对比, 绘制两者的散点图 (图 2) 并计算 3 种反演算法的 误差 (表 3). 为了更好地说明 IRS 热红外数据反演水温 的精度, 将同期的 TERRA 卫星 MODIS 温度产品与实测 水温值进行对比. 从图 2 可见, 3 种算法反演的水温值与 实测值非常接近, 两者的样点都在 1: 1直线附近, 与实测 水温的相关系数都在 0.9 以上, 以单窗算法的相关性最 高, 相关系数为 0.989 , 辐射传输模型法其次, 相关系数为 0.977 , 普适性单通道算法的相关性最低, 为 0.917 . 相比 之下, MODIS 温度产品的样点偏离 1:1直线的较多, 与实 测水温的相关系数为 0.935 . 从散点图可以看出, 3 种算 法从 IRS 热红外数据中反演的水温值与实测值有很好的 吻合度.

表 3 给出了 IRS 热红外数据反演水温的误差指标,

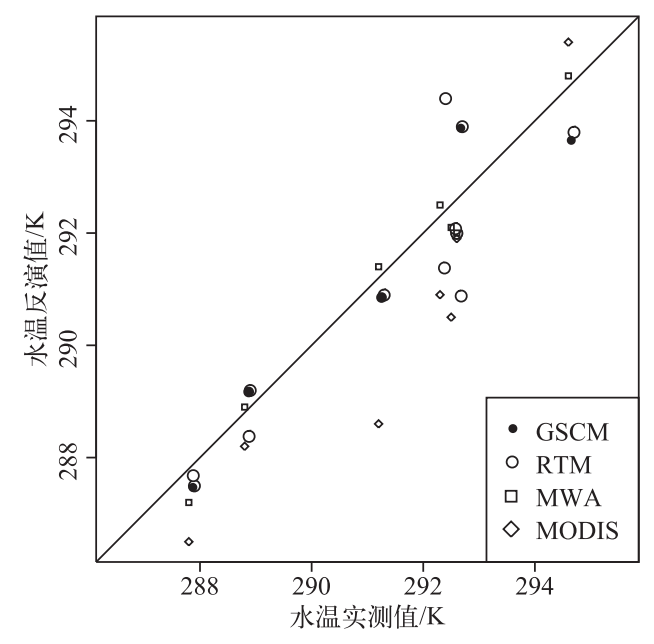

图 2 水温反演值与实测值的对比散点图

Fig. 2 Scatter plot between retrieved WST and measured one 平均偏差是各样点反演值与实测值之差的平均值, 平均 偏差越接近为 0 说明反演的系统误差越小, 大于 0 则说明反演值比实测值整体偏高, 否则相反; 而均方根误 差和平均相对误差反映了各样点反演值与实测值之间的相对差异, 其数值越低说明反演的误差越小、精度 越高. 与 MODIS 温度产品相比, 3 种算法从 IRS 热红外数据中反演水温的各项误差都明显低于 MODIS 数 据, 说明 HJ-1B 卫星获取的 IRS 数据质量是可靠的, IRS 热红外数据在湖泊水温遥感监测具有一定优势. 对 于反演水温的 3 种算法, 单窗算法和辐射传输模型法得到的平均偏差为负值, 说明这两种方法反演水温时 总体上会偏低,而普适性单通道算法的平均偏差为正值, 反演水温时会偏高, 3 种算法中以单窗算法的平均 偏差最接近 0 , 说明该方法反演水温的系统误差最小. 普适性单通道算法的均方根误差为 $1.001 \mathrm{~K}$, 其他两种 算法的均方根误差都小于 $1.001 \mathrm{~K}$; 而 3 种算法的平均相对误差都在 $0.3 \%$ 以下, 总体而言以单窗算法的误差 最小, 辐射传输模型法其次, 普适性单通道算法的误差最大.

分析 3 种算法产生的误差原因: 单窗算法反演水温时需要确定的参数有水的发射率、大气透过率和大 气平均作用温度, 辐射传输模型法的参数为发射率、大气透过率和大气上行热辐射亮度, 而普适性单通道算 法的参数仅为大气水汽量. 通过对比 ASTER 光谱数据库中自来水、蒸馏水和海水的光谱发现, 水的发射率 很稳定, 3 种水的发射光谱几乎是一致的. 由此可见,水的发射率对单窗算法和辐射传输模型法造成的误差 可以忽略. 大气透过率和大气上行热辐射亮度由辐射传输模型 MODTRAN 模拟得到, 尽管 LOWTRAN 和 MODTRAN 在海表温度和地表温度算法开发中被广泛应用, 但它们在大气窗口 3.4 4.1 和 8 13 $\mu \mathrm{m}$ 内模拟 的误差为 $0.5 \% \sim 2.0 \%$; 另外, 由于不同大气模型的差异, 热红外波段大气透过率在大气窗口内的误差达 6\%, 而在大气窗口边缘甚至超过 $30 \%{ }^{[20-21]}$. 因此大气透过率和大气上行热辐射亮度由 MODTRAN 模拟时带来了 不同程度的误差, 而在单窗算法中大气平均作用温度 $T_{\mathrm{a}}$ 根据探空资料由地面到高空 $200 \mathrm{pha}$ 之间每一层的 大气温度与水汽量加权平均计算得到 ${ }^{[22]}$, 相对于模拟的大气上行热辐射亮度, 大气平均作用温度能更准确 地反映大气自身热辐射情况, 因此单窗算法的反演精度比辐射传输模型法更高. 普适性单通道算法在反演 水温时直接将水体看作黑体, 而 IRS 热红外通道实际计算的水发射率为 0.9871 , 高估的发射率给水温反演带 来了误差, 因为赵利民等在分析 $\mathrm{HJ}-1 \mathrm{~B} / \mathrm{IRS}$ 热红外数据反演地表温度误差时发现, 在典型情况地表发射率 
估算误差为 0.01 时就能导致 $0.5 \mathrm{~K}$ 以上的反演误差 ${ }^{[23]}$; 另外,普适性单通道算法在温度反演时将大气透过 率和大气自身的热辐射都表示为水汽量的函数,龚绍琦等研究表明, 除了水汽量外,观测天顶角、能见度、气 溶胶类型都会影响热红外波段大气透过率 ${ }^{[19]}$; 大气自身的热辐射亮度还与大气温度有关. 普适性单通道算 法中的参数估计给反演结果带来了较大误差,因此该算法水温反演的精度相对较低.

表 3 水温反演算法的误差统计

Tab.3 Statistical errors of WST retrieval algorithms

\begin{tabular}{lcccc}
\hline 算法 & 平均偏差 $/ \mathrm{K}$ & 均方根误差 $/ \mathrm{K}$ & 平均相对误差 $/ \%$ & 相关系数 \\
\hline 普适性单通道算法 & 0.171 & 1.001 & 0.284 & 0.917 \\
辐射传输模型法 & -0.771 & 0.921 & 0.264 & 0.977 \\
单窗算法 & -0.129 & 0.380 & 0.113 & 0.989 \\
MODIS 温度产品 & -1.114 & 1.507 & 0.461 & 0.935 \\
\hline
\end{tabular}

\section{2 太湖水温反演结果的统计分析}

为了进一步探讨 3 种算法反演太湖水温的整体效果, 以 2009 年 4 月 21 日的数据为例进行说明, 分别对 3 种算法反演的太湖水温以及 MODIS 产品的太湖水温做直方图 (图 3), 统计其特征参数: 水温的最小值、最大 值、平均值、标准差、偏度和峰度 (表 4). 为了剔除图像的异常值, 这里的水温最小值和最大值不是图像上的极 值, 而是直方图左右两侧拐点位置的值, 以此对比分析不同算法对 IRS 热红外数据反演太湖水温的整体效果.
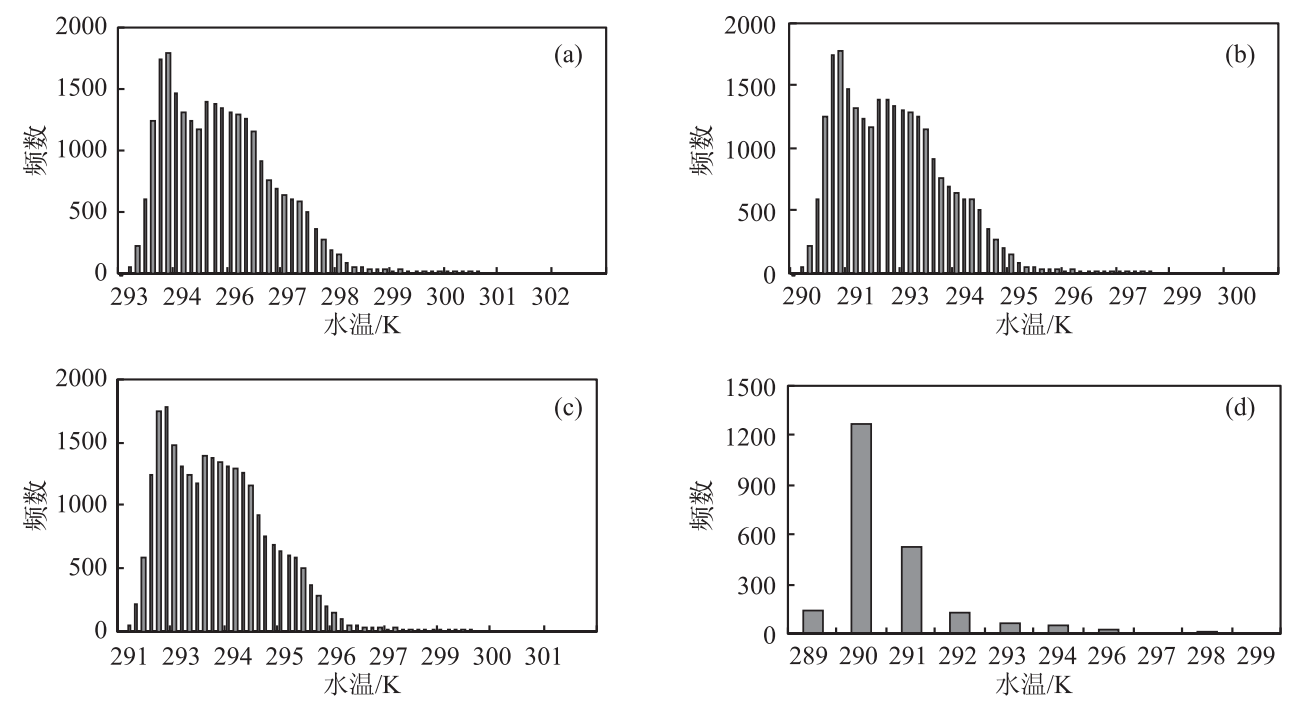

图 32009 年 4 月 21 日不同算法反演太湖水温的统计直方图 ( $\mathrm{a} \sim \mathrm{c}$ 分别为普适性单通道算法、辐射传输模型法和单窗算法, $d$ 为 MODIS 温度产品)

Fig.3 Histogram of WST in Lake Taihu retrieved by different algorithms on April 21, 2009 ( $\mathrm{a}-\mathrm{c}$ stand for GSCM, RTM and MWA, d stands for MODIS temperature product)

由图 3 和表 4 可见, 尽管 3 种算法得到太湖水温直方图形态相似, 但统计特征值均不同. 最小值、最高值和 平均值可以反映遥感图像水温值的动态范围和整体状况, 普适性单通道算法得到的水温最小值、最高值和平 均值最高, 其次是单窗算法, 再次是辐射传输模型法, MODIS 产品的温度最低. 这与前面分析的结果是一致的, 普适性单通道算法反演的结果要高于实测值, 而其它算法都低于实测值. 标准差整体反映了遥感图像上各像 元的水温值相对于平均值的离散程度, 相对于 MODIS 产品, 3 种算法反演水温得到的标准差都比较小, 其值在 1.175 1.231 之间, 说明水温反演值的离散程度较低. 偏度 (skewness) 和峰度 (kurtosis) 分别反映图像直方图分 布的扁平程度和形状大小. 偏度 $\alpha$ 是用于衡量直方图分布的不对称程度或偏斜程度的指标. 当 $\alpha=0$ 时为正态 
分布; 当 $\alpha>0$ 时为正偏态, 即位于均值右边的数据比左边的少, 直观表现为右边的尾部要比左边的尾部长; 否 则相反. 3 种算法反演的水温偏度都在 0.981 以上, 说明图像直方图呈正偏态, 图像上低于均值的像元数较多、 而高于均值的像元相对要少, 3 种算法反演的水温正偏态势依次是单窗算法、普适性单通道算法和辐射传输模 型法. 峰度 $\beta$ 是用于衡量图像直方图分布的集中程度或分布曲线的尖峭程度的指标, 当峰度 $\beta=0$ 时为正态分 布; $\beta>0$ 时, 表示分布比正态分布更集中在平均值周围,分布呈尖峰状态; $\beta<0$ 时,表示分布比正态分布更分散, 分布呈低峰态. 3 种算法反演的水温峰度都在 2 以上, 说明图像各像元的水温值更集中在平均值周围, 而这种 密集程度依次为: 单窗算法、普适性单通道算法和和辐射传输模型法. 由于 MODIS 数据的空间分辨率低,太湖 MODIS 水温产品的像元数少, 温度值的离散程度高, 直方图分布呈正偏态、尖峰状态更强.

表 42009 年 4 月 21 日不同算法反演太湖水温的统计结果

Tab.4 Statistical results of WST in Lake Taihu retrieved by different algorithms on April 21, 2009

\begin{tabular}{lcccccc}
\hline 算法 & 最小值/K & 最大值/K & 平均值/K & 标准差/K & 偏度 & 峰度 \\
\hline 普适性单通道算法 & 293.39 & 298.36 & 295.41 & 1.175 & 0.985 & 2.033 \\
辐射传输模型法 & 290.40 & 295.61 & 292.50 & 1.227 & 0.981 & 2.006 \\
单窗算法 & 291.56 & 296.78 & 293.67 & 1.231 & 0.992 & 2.069 \\
MODIS 温度产品 & 288.58 & 297.97 & 290.45 & 1.438 & 2.238 & 6.652 \\
\hline
\end{tabular}

\section{3 太湖水温反演结果的空间特征分析}

为了更直观地了解 3 种算法从 IRS 热红外数据中反演的太湖水温状况, 以 2009 年 4 月 21 日的数据为例, 分别绘制出太湖水温的空间分布图 (图 4), 并对水温空间分布图在 288 300 K 范围内按等间隔分级, 划出 288 291、291 294、294 297 和 297 300 K 4 级, 统计每种算法在每个温度等级内所占的面积(表 5). 由 IRS 热红外 图像统计的太湖水域总面积约为 $2350.00 \mathrm{~km}^{2}$, 而 MODIS 图像统计的水域面积为 $2240.00 \mathrm{~km}^{2}$, MODIS 图像统 计的水域面积偏低主要是由于其空间分辨率低,较小的水域未能划分出来. 从表 5 可见,普适性单通道算法反 演的水温主要分布在 294 297 K 之间, 其水域面积为 $1934.19 \mathrm{~km}^{2}$, 占太湖总面积的 $82.3 \%$, 而水温在 291 294 和 $297 \sim 300 \mathrm{~K}$ 的区域面积分别为 190.89 和 $224.91 \mathrm{~km}^{2}$, 占总面积的 $8.1 \%$ 和 $9.6 \%$. 辐射传输模型法反演的水 温主要分布在 291 294 K 之间, 其水域面积为 $1875.06 \mathrm{~km}^{2}$, 占总面积的 79. 8\%, 而水温在 288 291、294 297 和 $297 \sim 300 \mathrm{~K}$ 的区域面积分别为 $190.89 、 271.80$ 和 $12.24 \mathrm{~km}^{2}$, 占总面积的 $8.1 \% 、 11.6 \%$ 和 $0.5 \%$. 单窗算法反 演的水温主要分布在 $291 \sim 294 \mathrm{~K}$ 之间, 其水域面积为 $1461.42 \mathrm{~km}^{2}$, 占总面积的 $62.2 \%$, 其次是 $294 \sim 297 \mathrm{~K}$ 之 间, 水域面积为 $862.92 \mathrm{~km}^{2}$, 占总面积的 $36.7 \%$, 而水温在 $297 \sim 300 \mathrm{~K}$ 之间的水域面积则有 $25.65 \mathrm{~km}^{2}$, 占总面 积的 $1.1 \%$. 而 MODIS 温度产品的水温分布在 $288 \sim 291 \mathrm{~K}$ 之间, 水域面积为 $1942.00 \mathrm{~km}^{2}$, 占总面积的 $86.7 \%$, 水温大于 $291 \mathrm{~K}$ 的区域仅为 $298.00 \mathrm{~km}^{2}$, 占总面积的 $13.3 \%$. 由此可见, 不同算法反演的水温在空间上存在明 显的差异性; 普适性单通道算法反演的水温仍然最高, 其次是单窗算法, 再次是辐射传输模型法, 而 MODIS 温 度产品最低, 这与前面分析的结果是一致的. 但无论是 3 种算法从 IRS 热红外数据反演的水温, 还是 MODIS 产 品的水温, 由于受陆地温度的影响, 都是太湖水陆交界位置的水温较高. 另外, 太湖南部水温要比北部水温高, 南部水域如胥口湾、东太湖、夹浦、小梅口、新塘港, 特别是西山岛西部区域的水温较高, 而北部水域如贡湖湾、 梅梁湾、竺山湾、大浦港的水温相对较低, 这是因为每年 11 月至翌年 4 月正值太湖枯水季, 太湖南部地区气温 比北部地区高, 而水体吸热快、散热慢, 气温影响着水温的变化. 这一现象正好与陈绍良等 ${ }^{[24]}$ 对太湖水体温度 时空变化规律的研究结果相吻合. 由此可见,利用 3 种算法从 HJ-1B 卫星 IRS 热红外数据反演的水温能真实 地反映太湖水温的空间分布特征, 而 MODIS 产品的水温相对较低.

\section{3 结论}

利用 6 景过境太湖的 HJ-1B/IRS 热红外影像, 分别采用普适性单通道算法、辐射传输模型法和单窗算 法反演了太湖水温, 并与实测水温和同期的 TERRA/MODIS 温度产品进行对比, 结果表明:

1) 利用 3 种算法从 IRS 热红外数据中反演太湖水温的均方根误差在 $1.001 \mathrm{~K}$ 以内, 而 MODIS 温度产品的 误差为 $1.507 \mathrm{~K}$, 说明 $\mathrm{HJ}-1 \mathrm{~B}$ 卫星 IRS 热红外数据在湖泊水温遥感监测中具有更好的性能优势和反演精度. 
表 52009 年 4 月 21 日不同算法反演太湖不同水温区域面积 $\left(\mathrm{km}^{2}\right)$ 统计

Tab.5 Zonal statistics for each WST in Lake Taihu class by different algorithms on April 21, 2009

\begin{tabular}{lccccc}
\hline 算法 & $288 \sim 291 \mathrm{~K}$ & $291 \sim 294 \mathrm{~K}$ & $294 \sim 297 \mathrm{~K}$ & $297 \sim 300 \mathrm{~K}$ & 总面积 \\
\hline 普适性单通道算法 & 0.00 & 190.89 & 1934.19 & 224.91 & 2349.99 \\
辐射传输模型法 & 190.89 & 1875.06 & 271.80 & 12.24 & 2349.99 \\
单窗算法 & 0.00 & 1461.42 & 862.92 & 25.65 & 2349.99 \\
MODIS 温度产品 & 1942.00 & 201.00 & 85.00 & 12.00 & 2240.00 \\
\hline
\end{tabular}
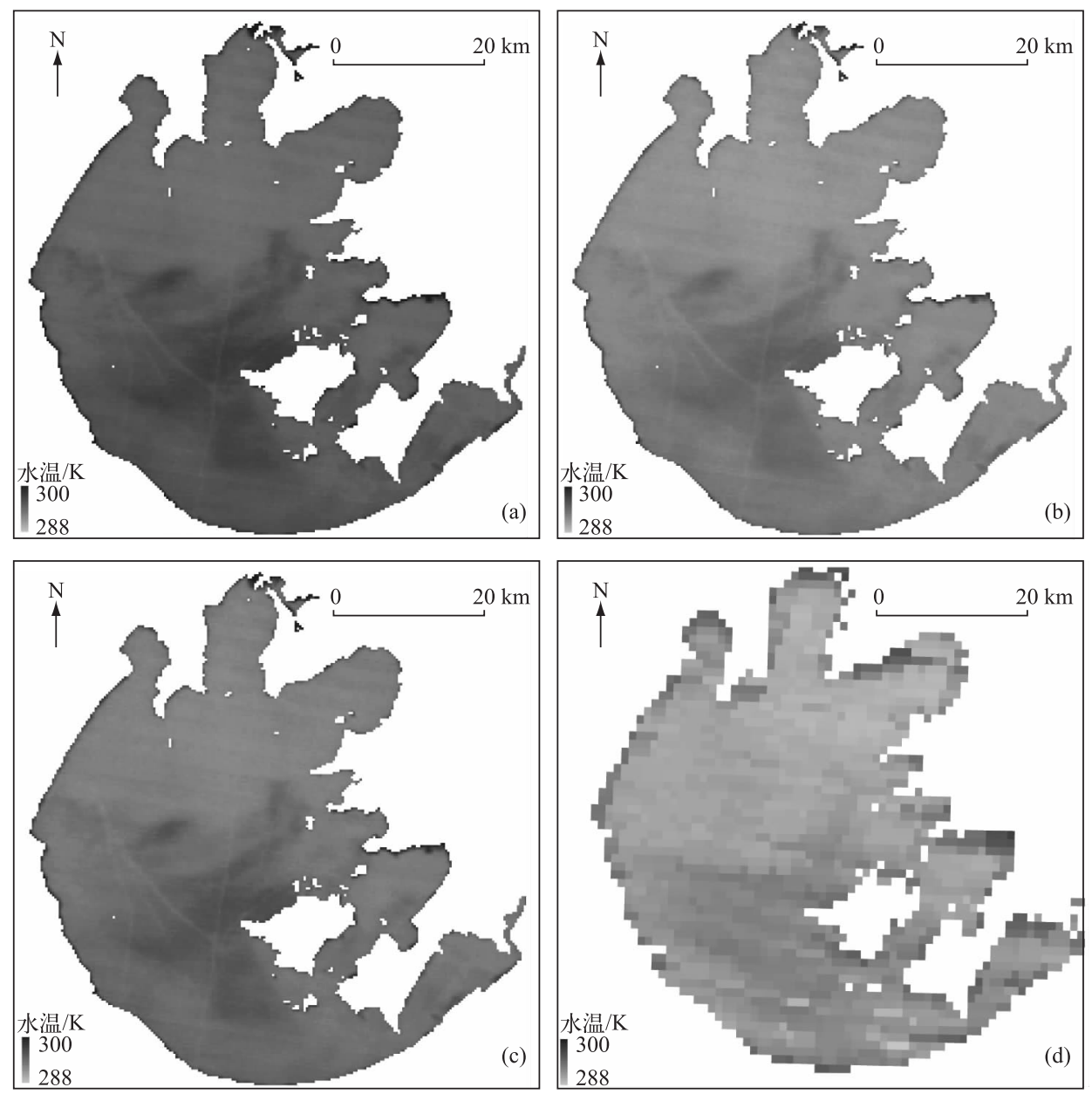

图 42009 年 4 月 21 日不同算法反演太湖水温的空间分布

( a c 分别为普适性单通道算法、辐射传输模型法和单窗算法, $d$ 为 MODIS 温度产品)

Fig.4 Spatial distribution of WST in Lake Taihu retrieved by different algorithms on April 21, 2009 ( $\mathrm{a}-\mathrm{c}$ stand for GSCM, RTM and MWA, d stands for MODIS temperature product)

2) 从平均偏差来看, 普适性单通道算法反演太湖水温时会偏高, 而辐射传输模型法和单窗算法则会偏 低. 3 种算法以单窗算法的反演精度最高, 辐射传输模型法其次, 再次为普适性单通道算法, 这是算法中不同 参数估算误差导致的.

3) 对太湖水温反演图像的直方图统计表明, 3 种算法反演的水温图像上低于平均值的像元数较多、高于 
均值的像元相对较少, 而且图像各像元的水温值更集中在平均值周围. 水温图像的空间特征分析表明, IRS 热红外数据反演的水温能真实地反映太湖水温的分布状况.

\section{4 参考文献}

[ 1 ] 秦伯强, 王小冬, 汤祥明等. 太湖富营养化与蓝藻水华引起的饮用水危机一一原因与对策. 地球科学进展, 2007, $22(9)$ : 897-907.

[ 2 ] 马荣华, 孔繁翔, 段洪涛等. 基于卫星遥感的太湖蓝藻水华时空分布规律认识. 湖泊科学, 2008, 20(6): 687-694. DOI $10.18307 / 2008.0605$.

[ 3 ] 段洪涛, 张寿选, 张渊智. 太湖蓝藻水华遥感监测方法. 湖泊科学, 2008, 20 (2)：145-152. DOI 10.18307/ 2008.0202.

[ 4 ] 王得玉, 冯学智, 周立国等. 太湖蓝藻爆发与水温的关系的 MODIS 遥感. 湖泊科学, 2008, 20(2): 173-178. DOI $10.18307 / 2008.0206$.

[ 5 ] 姜 晟, 张 咏, 蒋建军等. 基于 MODIS 数据的太湖蓝藻变化与水温关系研究. 环境科技, 2009, 22(6): 28-31.

[6] 任 健, 何 浪, 秦铭荣等. 太湖蓝藻生长发育与水温的关系. 中国农业气象, 2009, 30(增 1):126-129.

[ 7 ] 陈宇炜, 秦伯强, 高锡云. 太湖梅梁湾藻类及相关环境因子逐步回归统计和蓝藻水华的初步预测. 湖泊科学, 2001, 13(1) : 63-71. DOI 10.18307/2001.0110.

[8] 袁琳娜, 杨常亮, 李晓铭等. 高原深水湖泊水温日成层对溶解氧、酸碱度、总磷浓度和藻类密度的影响: 以云南阳 宗海为例. 湖泊科学, 2014, 26(1): 161-168. DOI 10.18307/2014.0120.

[9] 段四波, 阎广建, 钱永刚等. 利用 HJ-1B 模拟数据反演地表温度的两种单通道算法. 自然科学进展, 2008, 18 (9) : 1001-1008.

[10] Zhou J, Zhan WF, Hu DY et al. Improvement of mono-window algorithm for retrieving land surface temperature from HJ1B satellite data chin. Chinese Geographical Science, 2010, 20(2) : 123-131.

[11] 赵少华, 秦其明, 张 峰等. 基于环境减灾小卫星 (HJ-1B) 的地表温度单窗反演研究. 光谱学与光谱分析, 2011, 31(6) : 1552-1556.

[12] Ouyang XY, Li J, Pan YQ et al. Retrieval of land surface temperature over the Heihe River basin using HJ-1B thermal infrared data. Remote Sensing, 2015, 7: 300-318.

[13] Hu K, Chen F, Liang SW. Application of HJ-1B data in monitoring water surface temperature. Procedia Environmental Sciences, 2011, (10): 2042-2049.

[14] Jiménez-Muñoz JC, Sobrino JA. A generalized single-channel method for retrieving land surface temperature from remote sensing data. Journal of Geophysical Research Oceans, 2003, 108(D22) : 4688-4696.

[15] 覃志豪, Zhang MH, Karnieli A 等. 用陆地卫星 TM6 数据演算地表温度的单窗算法. 地理学报, 2001, 56(4): 456-466.

[16] 张 勇, 李 元, 戎志国等. 利用大洋浮标数据和 NECP 再分析资料对 FY-2C 红外分裂窗通道的绝对辐射定标. 红外与毫米波学报, 2009, 28(3): 188-194.

[17］孟凡斌, 郑 丽. 基于 LOWTRAN 7 的红外大气透过率计算方法.光电技术应用, 2009, 24(3): 29-33.

[18］覃志豪, Li WJ, Zhang MH 等. 单窗算法的大气参数估计方法. 国土资源遥感, 2003, 2: 37-43.

[19] 龚绍琦, 孙海波, 王少峰等. 热红外遥感中大气透过率的研究 (一) : 大气透过率模型的构建. 红外与激光工程, 2015, 44(6) : 1692-1698.

[20] Wan ZM, Dozier J. A generalized split-window algorithm for retrieving land-surface temperature from space. IEEE Transactions on Geoscience and Remote Sensing, 1996, 34(4) : 892-905.

[21] Wan ZM, Dozier J. Effects of temperature-dependent molecular absorption coefficients on the thermal infrared remote sensing of the earth surface. Proc. IGARSS'92, 1992: 1242-1245.

[22] Sobrino J, Coll C, Caselles V. Atmospheric correction for land surface temperature using NOAA-11 AVHRR channels 4 and 5. Remote Sensing of Environment, 1991, 38: 19-34.

[23] 赵利民, 余 涛, 田庆久等. HJ-1B 热红外遥感数据陆表温度反演误差分析. 光谱学与光谱分析, 2010, 30(12): 3359-3362.

[24］陈绍良, 陆建伟, 沈建强. 太湖水体温度时空变化规律的初步研究. 江苏水利, 2009, 3: 38-39. 